\title{
Análise comparativa entre a vascularização arterial ventricular e do nó sinoatrial em corações de cães ${ }^{1}$
}

\author{
Caio Biasi ${ }^{2}$, Vicente Borelli ${ }^{3}$, Rodrigo F. Prazeres ${ }^{4}$, Phelipe O. Favaron ${ }^{4 *}$, \\ Valdir Pavanelo Junior ${ }^{4}$, Thiago P.A. Aloia ${ }^{5}$ e Pedro P. Bombonato ${ }^{3}$
}

\begin{abstract}
Biasi C., Borelli V., Prazeres R.F., Favaron P.O., Pavanello Junior V., Aloia T.P.A. \& Bombonato P.P. 2013. [Comparative analysis between both ventricular and sinoatrial node arterial vascularization in heart of dogs.] Análise comparativa entre a vascularização arterial ventricular e a do nó sinoatrial em corações de cães. Pesquisa Veterinária Brasileira 33(1):111-114. Setor de Anatomia dos Animais Domésticos e Silvestres, Faculdade de Medicina Veterinária e Zootecnia, Universidade de São Paulo, Av. Prof. Dr. Orlando Marques de Paiva 87, São Paulo, SP 05508-030, Brazil. E-mail: phelipe.favaron@yahoo.com.br

We analyzed 40 hearts of adult dogs, males and females of different ages, without cardiac disease. The hearts had the coronary arteries inject using Neoprene Latex 450, colored with red pigment, and which was then dissected. In the ventricular vascularization the left coronary artery was predominant and gave rise to the paraconal interventricular and subsinuous branches. The region occupied by the sinoatrial node was most frequently (17 times, $42.5 \%$ ) in dependence of the left atrial proximal branch or in the collateral branch of that vessel, coming from the left circumflex branch, or this vessel was associated with the right atrial distal branch (8 times, 20\%). With less frequency (14 times, 30\%), in the area occupied by the sinoatrial node we found only the collaterals of the right circumflex branch, only the right atrial distal branch (10 times, $25 \%$ ), only the right atrial proximal branch (3 times, 7.5\%) or exclusively the right atrial intermediary branch (once, 2.5\%). In just one case (once, $2.5 \%$ ) in the area of the sinoatrial node we observed only the collateral of the left circumflex branch, i.e. the left atrial distal branch. According to our results we concluded that in this specie there is no relationship between the type of ventricular vascularization and irrigation of the sinoatrial node. In doing so, to consider just the ventricular branches is not sufficient for a clinical and surgical applied understanding, since the atrial branches make an important contribution to the sinoatrial node vascularization.
\end{abstract}

INDEX TERMS: Canines, cardiac conduction system, heart vascularization.

RESUMO.- Utilizamos nesta pesquisa 40 corações de cães adultos, machos e fêmeas, de idades variadas, que não portavam nenhuma afecção cardíaca. Os corações tiveram as artérias coronárias injetadas, separadamente, com Neopre-

\footnotetext{
${ }^{1}$ Recebido em 5 de outubro de 2012.

Aceito para publicação em 10 de dezembro de 2012.

${ }^{2}$ Instituto de Ciências da Saúde da Universidade Paulista (Unip), Rua Doutor Bacelar 1212, Vila Clementino, São Paulo, SP 04026-002, Brasil.

${ }^{3}$ Departamento de Cirurgia, Faculdade de Medicina Veterinária e Zootecnia (FMVZ), Universidade de São Paulo (USP), Av. Prof. Orlando Marques de Paiva 87, Cidade Universitária, São Paulo, SP 05508-900.

${ }^{4}$ Pós-graduando em Anatomia dos Animais Domésticos e Silvestres, Departamento de Cirurgia, FMVZ-USP, São Paulo, SP. *Autor para correspondência: phelipe.favaron@yahoo.com.br

${ }^{5}$ Pós-Doutorando em Neuroimunomodulação, Departamento de Patologia, FMVZ-USP, São Paulo, SP.
}

ne Látex 450, corado com pigmento vermelho, e posteriormente dissecados. Em todas estas preparações verificamos que na vascularização dos ventrículos predominava a artéria coronária esquerda que fornecia os ramos interventriculares paraconal e subsinuoso. Já, a região ocupada pelo nó sinoatrial ficava mais frequentemente (17 vezes, $42,5 \%$ ) na dependência do ramo proximal atrial esquerdo ou de colateral deste vaso, oriundo do ramo circunflexo esquerdo, ou deste vaso associado ao ramo distal atrial direito (8 vezes, $20 \%$ ), procedente do ramo circunflexo direito. Com menor frequência (14 vezes, 30\%), a área tomada pelo nó sinoatrial, encontramos apenas colaterais do ramo circunflexo direito, mais exatamente somente o ramo distal atrial direito (10 vezes, $25 \%$ ), apenas o ramo proximal atrial direito ( 3 vezes, $7,5 \%$ ) ou ainda exclusivamente o ramo intermédio atrial direito (1 vez, 2,5\%). Em um único caso (1 
vez, 2,5\%) no território do nó sinoatrial observamos apenas colateral do ramo circunflexo esquerdo, isto é o ramo distal atrial esquerdo. A análise destes resultados permite concluir, que nesta espécie não existe qualquer tipo de relação entre o tipo de vascularização dos ventrículos e a irrigação do nó sinoatrial. Sendo assim, considerar os ramos ventriculares isoladamente não é suficiente para um entendimento clínico-cirúrgico aplicado, uma vez que os ramos atriais apresentam uma importante contribuição para a vascularização do nó sinoatrial.

TERMOS DE INDEXAÇÃO: Caninos, sistema de condução cardíaco, vascularização cardíaca.

\section{INTRODUÇÃO}

Em 1904, Banchi descreveu três tipos de vascularização cardíaca estudando corações humanos. A vascularização do tipo esquerda, a qual se caracteriza pela artéria coronária esquerda emitir o ramo interventricular paraconal, o ramo circunflexo esquerdo e o ramo interventricular subsinuoso. A vascularização do tipo equilibrada, onde a artéria coronária direita fornece o ramo interventricular subsinuoso que divide com a artéria coronária esquerda a nutrição da face atrial ventricular do coração. E o último tipo de vascularização cardíaca, a direita, onde a artéria coronária direita, muito desenvolvida, depois de ceder o ramo interventricular subsinuoso, este avança para ganhar o sulco coronário esquerdo atingindo a margem ventricular esquerda do coração.

Na literatura que agora tivemos a oportunidade de examinar, notamos que diversos pesquisadores como Moore (1930), Lucke (1950), Andretto et al. (1973), Evans (1993) e Oliveira et al. (2011), que utilizando diferentes técnicas, analisaram e compararam, os critérios apresentados previamente por Banchi (1904), no que tange aos tipos de vascularização arterial em coração de cães a fim de melhor conhecer a predominância de uma das coronárias ou de ambas na nutrição do território ventricular.

Quanto ao nó sinoatrial, depois da descoberta de Keith e Flack em 1907, inúmeros pesquisadores (Lewis et al. 1910, Cohn et al. 1912, Condorelli 1929, Chiodi 1957, James 1962, Schaller 1962) estudaram os aspectos histológicos e a localização do nó sinoatrial, em corações de cães, informação indispensável para a realização da pesquisa ora proposta, sendo que dentre eles, James (1962) e Schaller (1962) esclareceram com exatidão que o nó sinoatrial encontra-se situado subepicardicamente na face atrial do átrio direito, em correspondência ao sulco terminal.

A importância funcional do nó sinoatrial tem levado inúmeros pesquisadores a se interessarem pelos aspectos morfológicos, funcionais e seu suprimento sanguíneo em diversas espécies animais como, por exemplo, em bovinos (Caetano 1982, Caetano et al. 1995, Ovèina 2002), em babuínos (Mandarim-de-Lacerda \& Hureau 1986), em suínos (Weaver et al. 1986, Severino et al. 2000, Vidotti et al. 2008), em ratos (Melo 2000, Melo et al. 2002) em humanos (Jatene et al. 1999, Berdajs et al. 2003), e em gatos (Biasi et al. 2012), e especialmente em cães (Pina et al. 1975, Fernandes Filho et al. 1975, Amaral et al. 1985, Randi 1988,
Ovèina 2002). Contudo, no que diz respeito à interdependência entre a irrigação do nó sinoatrial e a vascularização ventricular os dados ainda hoje são bastante escassos (Biasi et al. 2012).

Com base no que foi proposto, o presente trabalho avaliou a relação existente entre a vascularização arterial do nó sinoatrial e dos ventrículos de corações de cães, com o propósito de conhecer a participação real de cada uma das artérias coronárias na nutrição dos ventrículos e do nó sinoatrial propriamente dito.

\section{MATERIAL E MÉTODOS}

Foram utilizados 40 corações de cães adultos (17 machos e 23 fêmeas) com diferentes e não conhecidas idades, sem raça definida, procedentes do Hospital Veterinário da Universidade Paulista em São Paulo após eutanásia, os quais não eram portadores de afecções cardíacas. 0 experimento foi realizado seguindo os princípios éticos de experimentação animal da Comissão de Ética no uso de animais da Faculdade de Medicina Veterinária e Zootecnia da Universidade de São Paulo (Protocolo: 2341/2011).

Após isolamento do coração e retirada do saco pericárdico, cada coração foi lavado em água corrente e submetido à massagem manual para melhor esvaziamento das câmaras cardíacas e vasos sanguíneos. As artérias coronárias foram dissecadas e injetadas separadamente, com solução de Neoprene Látex 450 (Du Pont do Brasil S/A Indústrias Químicas, São Paulo), corado em vermelho ou preto.

Após as injeções com Neoprene Látex, os corações foram fixados em solução aquosa de formol a 10\% por no mínimo 72 horas para posterior dissecação da circulação ventricular e atrial. A nomenclatura adotada para identificação e descrição das artérias atriais baseou-se no proposto por Lucke (1950), com reparos de Habermehl (1959).

Fotografias foram tiradas (Sony MVC, CD500) para documentação do trabalho e estudos estatísticos foram efetuados, com o auxílio do Teste de Qui-quadrado com nível de significância de 5\% utilizando o programa Minitab 16.

\section{RESULTADOS}

A injeção de Neoprene Látex, seguida de dissecação, nos permitiu avaliar a vascularização ventricular, do nó sinoatrial e a relação entre a vascularização ventricular e do nó sinoatrial.

No tocante a vascularização ventricular dos corações agora estudados, em todos os espécimes, apresentou a do tipo esquerda, ou seja, a artéria coronária esquerda fornecia o ramo interventricular paraconal que nutria a face auricular dos ventrículos e o ramo circunflexo esquerdo que continuava diretamente como o ramo interventricular subsinuoso, irrigando a face atrial dos ventrículos cardía$\cos$ (Fig.1A).

Quanto à vascularização do nó sinoatrial, com grande frequência, em 17 casos (42,5\%) a região tomada pelo nó sinoatrial recebia exclusivamente o ramo proximal atrial esquerdo, diretamente ou através de colateral deste vaso (Fig.1B). Em boa parte dos corações estudados, 10 casos (25\%), a vascularização do aludido nó ficou na dependência do ramo distal atrial direito, diretamente ou mediante colateral, para atingir o sulco terminal (Fig.1C). Em 8 corações $(20 \%)$, a irrigação arterial do território ocupado 

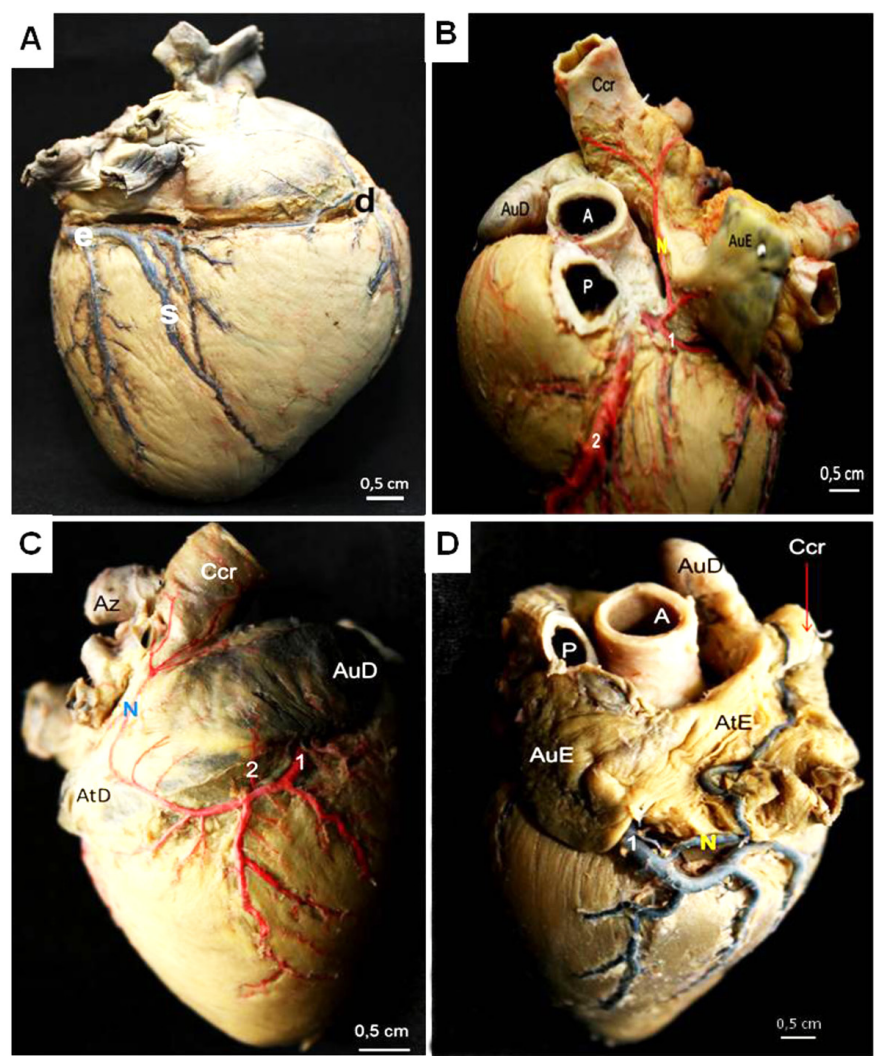

Fig.1. (A): Face atrial de coração de cão: ramo circunflexo direito (d), ramo circunflexo esquerdo (e), ramo interventricular subsinuoso (s). (B) Face auricular de coração de cão: aorta (A), aurícula direita $(\mathrm{AuD})$, aurícula esquerda $(\mathrm{AuE})$, veia cava cranial (Ccr), ramo proximal atrial esquerdo $(\mathrm{N})$, tronco pulmonar $(\mathrm{P})$, ramo circunflexo esquerdo (1), ramo interventricular paraconal (2). (C) Margem ventricular direita de coração de cão: átrio direito (AtD), aurícula direita (AuD), veia ázigos $(A z)$, veia cava cranial (Ccr), ramo distal atrial direito $(\mathrm{N})$, ramo circunflexo direito (1), ramo intermédio atrial direito (2). (D) Margem ventricular esquerda de coração de cão: aorta (A), átrio esquerdo (AtE), aurícula direita (AuD), aurícula esquerda (AuE), veia cava cranial $(\mathrm{Ccr})$, ramo distal atrial esquerdo $(\mathrm{N})$, tronco pulmonar (P), ramo circunflexo esquerdo (1).

pelo nó sinoatrial ficou na dependência de dois vasos, o ramo proximal atrial esquerdo e o ramo distal atrial direito. Com menor frequência, 3 corações (7,5\%), a vascularização do nó sinoatrial dependia do ramo proximal atrial direito, para diretamente ou mediante colateral, ganhar o sulco terminal. Duas modalidades diferentes de vascularização do nó sinoatrial foram encontradas analisando os 2 corações restantes, em uma delas o aludido nó recebia suprimento sanguíneo do ramo intermédio atrial direito e a outra o marcapasso cardíaco dependia do ramo distal atrial esquerdo (Fig.1D).

No que tange a relação entre as vascularizações arteriais ventricular e do nó sinoatrial, notamos que pode existir a participação exclusiva de vasos provenientes da artéria coronária esquerda na vascularização do nó sinoatrial, bem como da artéria coronária direita, ou ainda de ambas as artérias, caracterizando nestes espécimes grande diversidade de vasos encontrados ocupando total ou parcialmente a área destinada ao nó sinoatrial.
No respeitante à análise estatística não identificamos diferenças significativas entre os achados em relação ao sexo.

\section{DISCUSSÃO}

A vascularização ventricular arterial segundo conceitos de Banchi (1904) pode ser caracterizada por três tipos fundamentais, a esquerda, direita e equilibrada. Inúmeros pesquisadores (Moore 1930, Lucke 1950, Andretto et al. 1973, Evans 1993, Oliveira et al. 2011) estudando a vascularização ventricular em cães observaram que quase todos os espécimes analisados apresentavam a predominância da artéria coronária esquerda na irrigação dos ventrículos cardíacos, ou seja a vascularização era caracterizada como do tipo esquerda, fato este encontrado em todos os corações agora estudados.

A topografia e os aspectos histológicos do nó sinoatrial de cães sem raça definida (SRD), conhecimento indispensável para a presente pesquisa, depois dos achados de Keith \& Flack (1907), foi motivo de inúmeras pesquisas realizadas por Lewis et al. (1910), Koch (1911), Condorelli (1929), Chiodi (1957), cabendo, entretanto a James (1962) e Schaller (1962) descreverem com precisão a localização do aludido nó, identificado a ocupar posição subepicárdica, na junção da veia cava cranial com o átrio direito, em correspondência ao sulco terminal.

Nos corações agora examinados, verificamos que a vascularização da região tomada pelo nó sinoatrial ficou dependente do ramo proximal atrial esquerdo, o que não coincide com os resultados já descritos na literatura no que diz respeito à vascularização cardíaca, onde os mesmos, revelaram que na maioria das vezes, a vascularização do aludido nó ficou na dependência do ramo distal atrial direito tanto em cães SRD (Moore 1930 Kazzaz \& Shanklin 1950, Lucke 1950, James 1962, Fernandes Filho et al. 1975), assim como para cães das raças dobermann (Amaral et al. 1985) e boxer (Randi 1988).

De outra parte, a prevalência do ramo distal atrial direito como responsável mais frequente pela nutrição do nó sinoatrial (Moore 1930, Kazzaz \& Shanklin 1950, Lucke 1950, James 1962, Fernandes Filho et al. 1975, Amaral et al. 1985, Randi 1988), também foi vista nos corações agora examinados com significativa ocorrência, $25 \%$ dos casos analisados.

Já a associação do ramo proximal atrial esquerdo e ramo distal atrial esquerdo, no desempenho de tal função, aparece com marcada frequência em inúmeras publicações (Pianetto 1939, Fernandes Filho \& Borelli 1966, Andretto 1972, Fernandes Filho et al. 1975, Amaral et al. 1985, Randi 1988), fato este que também tivemos a oportunidade de visualizar em $20 \%$ dos casos estudados.

Ainda parte a circunstância de aparecer isoladamente o ramo proximal atrial direito (Fernandes Filho \& Borelli 1966, Randi 1988) ou do ramo intermédio atrial direito (Andretto 1972, Fernandes Filho et al. 1975, Randi 1988) em pequeno número de casos, fato este observado nos casos agora examinados.

Finalmente quanto a vascularização do nó sinoatrial, a diminuta ocorrência de uma só artéria com a responsabilidade de nutrir este tecido nodal, Andretto (1972) observou 
que o ramo intermédio atrial esquerdo ficou encarregado de nutrir tal componente anatômico, oportunidade que não tivemos de assinalar nos corações agora examinados, assim como todos os pesquisadores que trataram deste assunto, não evidenciaram a participação exclusiva do ramo distal atrial esquerdo, que observamos uma única vez.

0 que acabamos de descrever para os cães, também foi recentemente descrito para gatos (Biasi et al. 2012), de maneira que podemos concluir que não existe nenhum tipo de relação entre a vascularização da região ocupada pelo nó sinoatrial e a participação de cada uma das coronárias, ou de ambas, na vascularização ventricular. Além disso, baseando-se simplesmente no comportamento dos ramos ventriculares entendemos que fica prejudicada a interpretação do predomínio de uma das artérias coronárias ou equilíbrio delas na nutrição do coração, sem considerar a procedência dos vasos atriais destinados ao focado nó, pela sua reconhecida importância.

\section{CONCLUSÃO}

Os resultados encontrados permitem concluir que embora a contribuição ventricular tenha sido unicamente considerada quanto à vascularização do nó sinoatrial na literatura até o momento, a participação significativa de ramos atriais na vascularização do referido nó, em cães, deve ser também considerada não apenas devido à importância na perfeita manutenção do funcionamento cardíaco, mas também devido a sua importância num contexto clínico-cirúrgico.

\section{REFERÊNCIAS}

Amaral R.C., Borelli V. \& Didio L.J.A. 1985. The blood supply of the Sinuatrial Node of Dobermann Dogs. Arch. Ital. Anat. Embriol. 90(3):113-119.

Andretto R. 1972. Contribuição ao estudo da irrigação arterial do nó sinu-atrial, em cães da raça Pastor Alemão. Tese de Doutorado, Faculdade de Medicina, Unicamp, Campinas. 65p.

Andretto R., Borelli V. \& Fernandes Filho A. 1973. Sobre a origem do Ramus descendens subsinuosus em cães. Revta Fac. Med. Vet. USP 10:5-9.

Banchi A. 1904. Morfologia della arteriae coronariae cordis. Arch. Ital. Anat. Embriol. 3:87-164.

Berdajs D., Patonay L. \& Turina M.I. 2003. The clinical anatomy of the sinus node artery. Ann. Thorac. Surg. 76(3):732-736.

Biasi C., Borelli V., Benedicto H.G., Pereira M.R., Favaron P.O. \& Bombonato P.P. 2012. Análise comparativa entre os tipos de vascularização ventricular e do nó sinoatrial em gatos. Pesq. Vet. Bras. 32(1):78-82.

Caetano A.G. 1982. Contribuição ao estudo da irrigação arterial do Nodus sinuatrialis em bovinos da raça Gir. Dissertação de Mestrado, Escola Paulista de Medicina, USP, São Paulo. 63p.

Caetano A.G., Borelli V., Prates J.C. \& Didio L.J.A. 1995. The arterial supply of the Nodus sinuatrialis in bovine Gir. Braz. J. Morphol. Sci. 12(2):81-88.

Chiodi V. 1957. Le structure profonde del cuore in canidi. Atti Accad. Sci. Inst. Bologna 4:59-72.

Cohn A.E., Kessel L. \& Mason H.H. 1912. Observations on the functions of the sino-auricular node in the dog. Heart 3:311-338.

Condorelli I.L. 1929. Über die Bahnen der Reizleitung vom Keith Flack Knoten zu den Vorhöfen. Z. Ges. Exp .Med. 68:493-515.

Evans H.E. 1993. Miller's Anatomy of the Dog. $3^{\text {rd }}$ ed. W.B. Saunders, Philadelphia. 1113p.

Fernandes Filho A. \& Borelli V. 1966. Irrigação do nó-sinu-atrial em cães. Arqs Inst. Biológico, São Paulo, 33:119-124.
Fernandes Filho A., Borelli V., D’Errico A.A. \& Andretto R. 1975. Contribuição ao estudo da irrigação do nó sinu-atrial em cães da raça Pequinês. Revta Fac. Med. Vet. Zootec. USP 12:53-66.

Habermehl K.H. 1959. Die Blutgefässversorgung des Katzenherzens. Zbl. Vet. Med., Berlin, 6(1):655-680.

James T.N.1962. Anatomy of the sinus node of the dog. Anat. Rec. 143:251162.

Jatene M.B., Hervoso C.M., Terra R.M., Guimarães M.H., Monteiro R., Jatene F.B. \& Jatene A.D. 1999. Importância da anatomia da circulação coronária atrial na operação de Cox para controle da fibrilação atrial. Revta Bras. Cir. Cardiovasc. 14(1):32-38.

Kazzaz D. \& Shanklin W. 1950. The coronary vessels of the dog, demonstrated by collored plastic (vinyl acetate) injections and corrosion. Anat. Rec. 107:43-59.

Keith A. \& Flack M. 1907. The form and nature of muscular connections between the primary of the vertebrate heart. J. Anat. 41:172-189.

Koch W. 1911. Welche Bedeutung kommt dem Sinus-Knoten zu? Med. Klin. $7: 447-452$.

Lewis T., Oppenheimer B.S. \& Oppenheimer A. 1910. The site of origin of the mammalian heart-beat; the pecemaker in the dog. Heart 2:147-169.

Lucke R. 1950. Blutgefässversorgung des Hundeherzens. Inaugural Dissertation, Tierärztliche Hochschule Hannover, Hannover. 62p.

Mandarim-de-Lacerda C.A. \& Hureau U.J. 1986. Arterial vascularization of the sino-atrial and atrio-ventricular nodes and frequency of coronary artery branches in Papio erxleben, 1777. Folia Primatol., Basel, 46(2):6569.

Melo S.R. 2000. Estudo estereológico, histológico e ultra-estrutural do nó sinoatrial em ratos adultos e velhos. Tese de Doutorado, Instituto de Ciências Biomédicas, USP, São Paulo. 64p.

Melo S.R., Mandarim-de-Lacerda C.A. \& Sousa R.R. 2002. Características ultra-estruturais do nó sinoatrial de rato Wistar. Acta Scient. 24(3):681685.

Moore R.A. 1930. The coronary arteries of the dog. Am. Heart J. 5(6):743749.

Oliveira C.L.S., David G.S., Carvalho M.O., Dornelas D., Araújo S., Da Silva N.C., Ruiz C.R., Fernandes J.R. \& Wafae N. 2011. Anatomical indicators of dominance between the coronary arteries of dogs. Int. J. Morphol. 29(3):845-849.

Ovèina F. 2002. Vascularization of the sinoatrial segment in the heart conduction system in bovine and canine hearts. Med. Arch. 56(3):123125.

Pianetto M.B. 1939. The coronary arteries of the dog. Am. Heart J. 18:403410.

Pina J.A., Pereira A.T. \& Ferreira Ados S. 1975. Vascularisation arterielle du noeud sino-auricular du coeur chez le chien [Arterial vascularization of the sino-auricular node of the heart in dogs]. Acta Cardiol. 30(2):67-77.

Randi R.E. 1988. Contribuição ao estudo da irrigação do nó sinoatrial em cães da raça Boxer. Tese de Doutorado, Faculdade de Medicina Veterinária e Zootecnia, USP, São Paulo. 66p.

Schaller 0. 1962. Die arterielle Gefässversorgung des Erregungsleitungssystems des Herzens bei einigen Säugetieren. I. Die arterielle Gefässversorgung des Nodus sinu atrialis beim Hund (Canis familiaris). Morphol. Jb. 102(4):508-540.

Severino R.S., Carneiro e Silva F.O., Drummond S.S., Bombonato P.P. \& Alves F.P. 2000. Irrigação do nó sinoatrial em suínos da raça Piètrain. Braz. J. Vet. Res. Anim. Sci. 37(4):13-16.

Vidotti A.P., Agreste F.R., Bombonato P.B., Prado I.M.M. \& Monteiro R. 2008. Vascularização arterial da região do nó sinoatrial em corações de suínos: origem, distribuição e quantificação. Pesq. Vet. Bras. 28(2):113-118.

Weaver M.E., Pantely G.A., Bristow J.D. \& Ladley H.D. 1986. A quantitative study of the anatomy and distribution of coronary arteries in swine in comparison with other animals and man. Cardiovasc. Res. 20(12):907917. 